\title{
EDUCATION AND TRAINING Virtual reality and the transformation of medical education
}

\author{
Author: Jack Pottle ${ }^{\mathrm{A}}$
}

Medical education is changing. Simulation is increasingly becoming a cornerstone of clinical training and, though effective, is resource intensive. With increasing pressures on budgets and standardisation, virtual reality (VR) is emerging as a new method of delivering simulation. VR offers benefits for learners and educators, delivering cost-effective, repeatable, standardised clinical training on demand. A large body of evidence supports VR simulation in all industries, including healthcare. Though VR is not a panacea, it is a powerful educational tool for defined learning objectives and implementation is growing worldwide. The future of VR lies in its ongoing integration into curricula and with technological developments that allow shared simulated clinical experiences. This will facilitate quality interprofessional education at scale, independent of geography, and transform how we deliver education to the clinicians of the future.

KEYWORDS: Virtual reality, medical education, nursing education, simulation, innovation

\section{Introduction}

The pace of change in medical practice is relentless. The complex needs of an ageing population, the range of treatment options available, the interprofessional nature of care and the complexity of healthcare systems themselves are vastly different today than they were 20 years ago.

As such, how we prepare future clinicians for practice has had to adapt. It is no longer a question of whether an individual can retain or access facts, but how they use them, evaluate them and apply them to patient care.

There is therefore a move to replace rote learning with more clinically relevant and practical teaching. Problem-based learning, communication skills training and simulation-based learning have all entered curricula. With the increasing drive to provide clinical learning experiences, and the inherent difficulties in doing so, simulation in particular has gained momentum as a method of delivering experiential learning.

Simulation is an educational technique that involves creating situations that replicate real life, letting a learner act as they

Author: ${ }^{\mathrm{A}}$ co-founder and chief medical officer, Oxford Medical Simulation, London, UK would do in real life, then providing feedback and debrief on performance. Simulation is effective in many domains and has been found to be 'superior to traditional clinical education, producing powerful educational interventions that yield immediate and lasting results.'

However, while simulation is becoming central to healthcare education, it requires significantly more resources than traditional education. At a time when healthcare systems and educational institutions globally are struggling with growing demands and limited budgets, additional resources are hard to come by.

Fortunately, there has been a recent dramatic expansion in the ways in which we can deliver medical education. This has not only been through the internet and mobile devices, but through immersive technologies. These technologies - including augmented reality (AR) and virtual reality (VR) - can transform how we deliver educational experiences.

VR in particular has been adopted across medical and nursing fields. VR involves the user putting on a VR headset to become completely immersed in an interactive virtual environment. When used with appropriate educational software, this allows the user to learn from experience in the virtual world. This paper outlines what VR is; its strengths, its weaknesses, the evidence behind it, its use in practice and where the future lies.

\section{What is virtual reality?}

VR is the use of software to create an immersive simulated environment. Unlike traditional user interfaces, to experience VR, users put on head-mounted display (HMD) which places the user inside an experience, where they can engage with the environment and virtual characters in a way that feels real. VR has a unique power, more than any other technology that has ever existed, to make users believe they are in a different environment. This allows them to learn from experience as they would do in real life. ${ }^{2}$ This ability to deliver experiences on demand is where the power of VR lies.

\section{Screen-based learning}

Confusingly, screen-based learning has previously been referred to as 'virtual reality' in the medical literature. However, there is now an understanding that the value of virtual reality comes from immersion and the sense of presence - the feeling of 'being there' - that it generates. ${ }^{3,4}$ As such, only VR that is immersive - using headsets that completely block out the real world - is now referred to as 'virtual reality'. 


\section{0-video}

360 -video is a method of filming in 360 degrees to create a complete picture of the environment. To record 360-video, a camera is used that can film in every direction at once. Such recordings can then be viewed using a VR headset, allowing the viewer to feel like they are in the middle of the film. 360-video is a suitable medium if the aim is to provide the learner with a non-interactive experience of an environment. Examples of use include using 360-video to immerse patients in new virtual worlds to distract them during painful procedures.

However, 360-video is a largely passive experience. Viewers cannot interact realistically as the video is purely a linear recording, nor can they move realistically as the video is recorded from one location. This disconnect between the user's movement in the real world with their lack of movement in the virtual world can also lead to a sense of nausea in 360-video.

\section{Interactive VR}

By contrast, interactive VR involves a totally immersive, dynamic, adaptive, interactive world.

If you can imagine being in a highly realistic computer game, you will have some approximation of what VR feels like. In the context of medical training this can include virtual wards, interactive patients, colleagues and relatives, with interaction similar to the real world.

For example, in a scenario of a patient with chest pain presenting to the emergency department (ED), the learner can be in the virtual ED, moving and interacting with the virtual environment and patient as they would in real life. They can take a history, examine, investigate, diagnose and treat the patient. Family members and an interdisciplinary team can be added, with everything from patient observations to blood gases to realistic conversation adapting dynamically, as in real life. Patients can become confused, agitated and look physically unwell, while the bustle of a virtual hospital and emotional engagement with emergency scenarios and lifelike characters in real time builds a sense of stress.

The focus in such scenarios is on decision making, critical thinking and clinical reasoning, with scenarios being designed to replicate human interaction in the real world.

Once scenarios are completed, learners can receive virtual debriefing and view automatically-generated feedback on their performance. This feedback and debrief is central to the learning outcomes in any simulation, whether delivered in VR or through a manikin. In VR, feedback can be provided on technical and nontechnical skills carried out in the simulation relative to best practice. This allows learners to examine their performance in more detail and provides the opportunity for blended learning. It also facilitates peer learning as learners can share feedback with their colleagues and mentors as a basis for discussing specific learning points.

Companies, such as Oxford Medical Simulation, are already delivering such platforms globally, with scenarios covering medicine, nursing, paediatrics, psychiatry, and community health, with content expanding across fields.

\section{What can VR offer?}

VR offers distinct benefits for learners, faculty and the health system.

For learners, VR makes accessing clinical experiences simple. VR systems usually comprise a headset and laptop combination. They are commercially available, so are simple to setup, and designed for ease and safety of use. Many VR systems work with no faculty required. Learners can go to the VR system and take part in simulation whenever they like. This is not confined to large centres or high setup budgets so allows for much broader, flexible access. This flexibility of access allows the integration of simulationbased education into everyday practice. Simulation can become a regular occurrence around other learning activities - more like going to the gym than a one-off faculty-heavy training day. Vitally, VR scenarios are repeatable. This allows learners to make mistakes safely and then learn through deliberate practice to improve performance. This has been noted as one of the central features to successful simulation but one that cannot be accomplished with the space, time and faculty requirements of many simulation centres. ${ }^{5}$

In addition, the psychological safety, enjoyable nature and potential for gamification of VR encourages engagement and autonomous learning.

From an institutional standpoint, VR allows simulation to be delivered at reduced cost with fewer resources. The costs of physical simulation vary widely between institutions and defining the cost of physical simulation is complex. In medical education and simulation, costs are often difficult to assess and, when attempts are made, are frequently under-reported. ${ }^{6,7}$

Those studies that do attempt to define the cost of fully immersive medical simulation approximate that for one learner to lead one simulation scenario costs over $€ 200$; for example, McIntosh concludes 'Set up cost was US $\$ 876,485$ [ $€ 758,300$ ] (renovation of existing facility, equipment). Fixed costs per year totalled $\$ 361,425$ [£275,000]. Variable costs totalled $\$ 311$ [£237] per course hour' and Iglesias-Vázquez states that the 'cost of ALS [advanced life support] simulation for a 4 -day course is $€ 1,320$ [ $€ 1,140]$ per passed participant. $^{, 8,9}$

Virtual simulation costs often comprises hardware and software. High-end VR hardware costs approximately $£ 3,000$ for a setup (laptop and headset). Software costs depend on provider and quality of product, but is frequently under one-tenth of the cost of physical simulation independent of provider. ${ }^{10}$ As such, there are substantial setup and running cost savings to be made with virtual reality.

As well as financial savings, such technologies free up space and faculty time. Faculty do not necessarily need extra training to be able to use the VR equipment - most is commercially available hardware and intuitive software. Once running, some VR setups require a faculty member to be present, others do not, and faculty input is often dictated by specific use cases of the given VR system. VR can deliver the clinical scenario in a small space $(2 \times 2 \mathrm{~m})$ with under 5 minutes of setup. This simplicity of use allows other simulation activities to take place in a centre while VR simulation is occurring. This can include more faculty focus on advanced communication skills or in situ simulation, neither of which is wellsuited to VR.

Any virtual scenario should also be objective and standardised, ensuring consistent quality and adherence to protocols, so institutions can embed their latest protocols and ensure clinicians have practiced using them prior to seeing patients.

Additionally, many immersive systems allow the creation of bespoke simulation curricula to meet specific needs. These systems can also generate large amounts of performance data. This data is valuable for ensuring utilisation, encouraging learner engagement and for identifying struggling students who may benefit from further training. 
Finally, from a global health perspective, this reduction in cost and equity of access allows simulation to be distributed globally. This potential to democratise the availability of quality medical training makes VR an exciting prospect in healthcare training.

\section{What are the drawbacks?}

Despite the advantages, VR simulation is not a panacea. Rather, it is a tool used to accomplish a defined set of learning outcomes and should be deployed as such, integrated within an institution's curriculum and pedagogy to ensure effective use.

For example, VR is not suitable for every possible educational opportunity. It is not the best way to teach abdominal palpation; there is no need for complex immersion in this situation, just an accurate physical representation of an abdomen. The same applies for part task training, such as cannulation or many other procedural skills.

Virtual characters are often controlled by artificial intelligence (AI) systems. Though this is developing fast, it not yet suitable for certain learning objectives, such as breaking bad news. The complexities of language processing and facial expressions are, at present, best covered by a human rather than a virtual patient.

Educational barriers aside, there are difficulties with any new technology, implementing it requires faculty support. It can be difficult for faculty to engage more senior members, as they may see VR as a game, rather than an educational tool. However, many companies provide trial periods to allow educators to become comfortable with the technology, and fear of VR becomes less of an issue as its use becomes more widespread.

Key to the conversations that institutions have about VR is the acknowledgement that VR should not replace the expert educator. In much the same way that physical simulation should not replace clinical training, VR is just a technology to deliver a learning technique, in this case simulation. There are certain learning objectives that are best taught through physical simulation, there are others that are best taught through VR. Educators need to decide which objective they are looking to improve and determine the most appropriate method of delivery. With this approach, clinicians, universities and healthcare institutions can increase simulation delivery at reduced cost, while taking the burden off faculty and ensuring training quality.

\section{Does VR simulation work?}

Numerous lines of evidence demonstrate how VR and the immersion it offers deliver effective experiential learning. ${ }^{3,11}$ The efficacy of VR is evident in practice, already being a vital teaching tool in multiple fields, including aviation, oil, shipping and the military. ${ }^{12}$ In fact, the aviation industry credits VR-based simulation as a major contributor to a nearly $50 \%$ reduction in human error-related airline crashes since the 1970s. ${ }^{13}$

Studies in healthcare support the value of VR. For example, medical students demonstrate significantly higher knowledge gain when using an immersive environment rather than screen-based learning. ${ }^{4}$ Immersive VR has subsequently been widely adopted in surgical training where it has been shown to decrease injury, increase speed of operations and improve overall outcomes. It has now been absorbed into a large number of surgical programmes with excellent results. ${ }^{14}$

While medical and nursing fields have been slower to take up VR than surgeons, there is plenty of evidence available. For example,
VR can be used to train clinicians in complex procedures such as transvenous lead extraction, is effective in cardiopulmonary resuscitation training, can improve communication skills, enhance critical thinking and improve clinical decision making. ${ }^{15-19}$

VR has also been favourably tested against physical simulation. In one paper, 84 nursing students were randomised to either a virtual or physical nursing simulation. Learning transfer was the same in both, with no significant difference in performance between groups, but the simulation in VR group was found to be significantly cheaper. ${ }^{20}$

VR, therefore, has a number of benefits over traditional training and a solid evidence base across medical and nursing education. So how is it being used today?

\section{How is VR used in practice?}

VR simulation is used in medical and nursing schools as well as in postgraduate education around the world (Fig 1).

Practical implementation and curriculum integration vary depending on the VR platform and institutional need. See Box 1 for case studies on the University of Northampton and Oxford University Hospitals.

As well as hospitals and universities, VR systems are also being used across healthcare systems, with Health Education England, East of England supporting delivery of VR simulation across 18 NHS trusts from August 2019.

As such, VR simulation can fit around institutional needs as required. Though the specific examples mentioned here refer only to education and training, VR simulations are also being used in other areas. The standardised and objective nature of scenarios has allowed various institutions to implement assessment and recruitment programmes using VR. In recruitment, VR scenarios are being used as a proxy for clinical competency and form a basis for ongoing interviews. This facilitates recruitment locally as well as

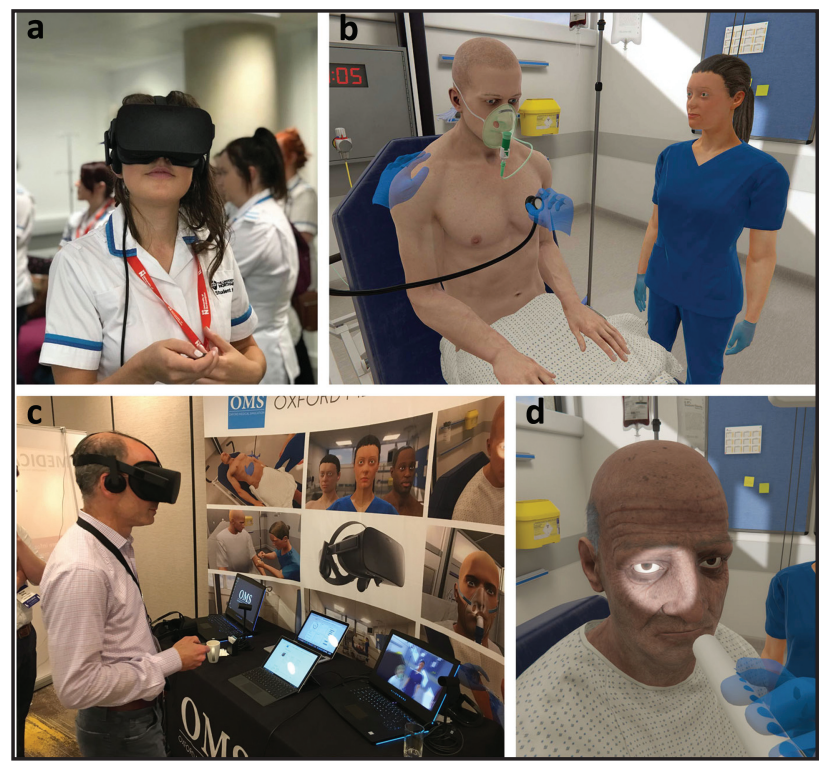

Fig 1. Virtual reality in use. a) Nursing student wearing a virtual reality headset. b) Performing a cardiac examination on a virtual patient. c) Projecting a virtual reality experience on multiple screens for group learning. d) Pupil responses to light in a virtual patient. 
Box 1. Case studies of universities using virtual reality simulation.

\section{University of Northampton}

The University of Northampton have created a virtual reality (VR) simulation suite for nursing students, with four sets of VR hardware and a large screen integrated in a physical simulation ward. This screen allows projection of what the learner is experiencing in VR onto the screen for group teaching. In small groups, learners take turns leading VR scenarios with real-time peer contribution, before doing a group debrief like physical simulation. This integration of VR within the simulation space ensures innovation complements existing educational structures and allows learners to practice simulation at scale while maintaining the value of peer support.

As noted by the nursing faculty:

Nurses require people skills, soft skills and clinical skills, and we needed to be able to train future nurses in a balanced way that caters to each of these skill sets. Technological developments are allowing us to do this in a safe and supportive learning environment, focusing on immediate feedback and the opportunity to repeat the scenarios and improve over time. VR simulation allows us to integrate theory into practice in a really meaningful manner, allowing students to progress throughout their academic careers.

\section{University of Oxford}

The University of Oxford are using VR simulation for their medical students and doctors working in the John Radcliffe Hospital. Rather than integrating VR in a defined simulation space, the faculty implemented mobile VR trolleys to transport their equipment wherever it is needed. Peer learning has been facilitated by groups of 'super-users' who introduce VR-naive students to the system. As a result of this, no faculty are required for use other than when they choose to review student progress or if the students approach them with clinical issues encountered in VR.

The faculty explained:

Simulation is a vital part of medical education and students just don't get to do it enough. Embedding VR simulation into what we do has enabled us to give a far greater number of learners access to simulation in a shorter space of time, and lets them do it as often as they like to transfer their knowledge to practice. It's encouraging to see how quickly our students have adopted the technology and I'm excited to see how they progress clinically as they use it more and more.

overseas, as the technology works in any setting and does not need expert faculty to run.

A number of institutions are also investigating VR from the standpoint of objective structured clinical examinations, as a method of decreasing the cost and increasing the objectivity of their assessment processes. These avenues remain at an early stage but are set to expand over the coming years.

In both assessment and recruitment situations, the utility of VR is in saving time, space, physical resources, need for expert faculty and removing geographical boundaries. In both cases however, the stakes are higher than in education and rigorous validation per institution becomes vital.

\section{Where does the future lie?}

As pressure to increase delivery of simulation continues, VR simulation will continue to expand. Rather than simulation being an occasional, faculty-led, day-long event, VR will allow simulation to be more like going to the gym. Learners will be able to do a scenario at the end of their shift or even at home, allowing continual improvement in performance to suit learner needs.

The standardisation and scoring possible with VR will make it commonplace in assessment and recruitment. In time, VR will become used routinely for continuing medical education and revalidation and become a benchmark to ensure clinical competency and patient safety across healthcare systems.

Then there are the technological advances. Increasing use of hand control (for complex procedural tasks) and voice control (for communication skills) are becoming viable. Haptics (the sense of touch in VR) will become increasingly used and all of these technological advances will become integrated within scenarios, blurring the lines between the real and the virtual.

The integration of AI will not only make interaction with virtual patients more realistic but allow increasingly in-depth analysis of clinical performance. AI can be used to tease out particular issues across large numbers of learners and offer dynamic, tailored scenarios to meet specific learning needs.

Most excitingly, although much current use focuses on individual learners in VR scenarios, multiplayer VR is becoming available. Multiplayer VR allows many disparate learners to see each other, talk to each other and interact with each other (and the patient) in the same virtual scenario.

This allows remote, collaborative learning in a real time clinical case - a paradigm that has never been possible before. With such systems, a doctor in Oxford can be seeing a virtual patient supported by a nurse in Delhi while being mentored by a professor in Baltimore.

This co-learning across cultures, essentially offering clinical experiences on demand, has the ability to revolutionise global healthcare education. The increased virtual clinical exposure that VR can offer, allowing an early focus on human factors and nontechnical skills, also has the ability to accelerate learning curves, and will contribute to the potential shortening of training timelines.

\section{Summary}

VR is already transforming medical education. It is helping to free learning from the classroom, allowing learners to apply their knowledge to practice and learn from mistakes. It focuses on improving competencies and places the emphasis on autonomous, blended learning, which is expected from the learners of today.

As VR continues to be implemented and integrated within curricula, its use will become mainstream. The ability for multiple learners to take part in truly interprofessional, completely life-like simulation which is not bound by geography, is set to change how we conduct medical and interprofessional education beyond recognition.

\section{References}

1 McGaghie WC, Issenberg SB, Petrusa ER, Scalese RJ. A critical review of simulation-based medical education research: 2003 2009. Med Educ 2010;44:50-63.

2 Bailenson JN. Experience on demand: what virtual reality is, how it works, and what it can do. New York: WW Norton, 2018. 
3 Makowski D, Sperduti M, Nicolas S, Piolino P. 'Being there' and remembering it: Presence improves memory encoding. Conscious Cogn 2017;53:194-202.

4 Gutiérrez F, Pierce J, Vergara VM et al. The effect of degree of immersion upon learning performance in virtual reality simulations for medical education. Stud Health Technol Inform 2007;125:155-60.

5 Issenberg SB, McGaghie WC, Petrusa ER, Lee Gordon D, Scalese RJ. Features and uses of high-fidelity medical simulations that lead to effective learning: a BEME systematic review. Med Teach 2005;27:10-28.

6 Walsh K, Jaye P. Simulation-based medical education: cost measurement must be comprehensive. Surgery 2013;153:302.

7 Zendejas B. Cost: The missing outcome in simulation-based medical education research: A systematic review. Surgery 2013;153:160-76

8 McIntish C. Simulation: What does it really cost? Simul Healthc 2006:1:109.

9 Iglesias-Vázquez J. Cost-efficiency assessment of Advanced Life Support (ALS) courses based on the comparison of advanced simulators with conventional manikins. BMC Emerg Med 2007;7:18.

10 Oxford Medical Simulation, 2019. https://oxfordmedicalsimulation. com [Accessed 09 September 2019].

11 Repetto C, Serino S, Macedonia M, Riva G. Virtual reality as an embodied tool to enhance episodic memory in elderly. Front Psychol 2016;7:1839.

12 Vozenilek J, Huff JS, Reznek M, Gordon JA. See one, do one, teach one: advanced technology in medical education. Acad Emerg Med 2004:11:1149-54.
13 Levin A. Fewer crashes caused by pilots. USA Today 2004. http://usatoday30.usatoday.com/news/nation/2004-03-01crash_x.htm [Accessed 12 April 2019].

14 Bric JD, Lumbard DC, Frelich MJ et al. Current state of virtual reality simulation in robotic surgery training: a review. Surg Endosc 2016;30:2169.

15 Maytin M, Daily TP, Carillo RG. Virtual reality lead extraction as a method for training new physicians: a pilot study. Pacing Clin Electrophysiol 2015;38:319-25.

16 Creutzfeldt J, Hedman L, Felländer-Tsai L. Cardiopulmonary resuscitation training by avatars: a qualitative study of medical students' experiences using a multiplayer virtual world. JMIR Serious Games 2016;4:e22.

17 Real FJ, DeBlasio D, Beck AF et al. A virtual reality curriculum for pediatric residents decreases rates of influenza vaccine refusal. Acad Pediatr 2017:17:431-5.

18 Burke SM. Cultivating critical thinking using virtual interactive case studies. J Pediatr Nurs 2017;33:94-6.

19 Harrington CM, Kavanagh DO, Quinlan JF et al. Development and evaluation of a trauma decision-making simulator in Oculus virtual reality. Am J Surg 2018;215:42-7.

20 Haerling KA. Cost-utility analysis of virtual and mannequin-based simulation. Simul Healthc 2018;13:33-40.

Address for correspondence: Dr Jack Pottle, Oxford Medical Simulation, 10 Old Town Hall, 19 Spa Road, London SE16 3SA, UK. Email: jack@oxfordmedicalsimulation.com 